\title{
The evolution of the accretion disk around 4U 1820-30 during a superburst
}

\author{
D.R. Ballantyne ${ }^{1}$ and T.E. Strohmayer ${ }^{2}$
}

\begin{abstract}
Accretion from a disk onto a collapsed, relativistic star - a neutron star or black hole - is the mechanism widely believed to be responsible for the emission from compact X-ray binaries. Because of the extreme spatial resolution required, it is not yet possible to directly observe the evolution or dynamics of the inner parts of the accretion disk where general relativistic effects are dominant. Here, we use the bright X-ray emission from a superburst on the surface of the neutron star $4 \mathrm{U} 1820-30$ as a spotlight to illuminate the disk surface. The X-rays cause iron atoms in the disk to fluoresce, allowing a determination of the ionization state, covering factor and inner radius of the disk over the course of the burst. The time-resolved spectral fitting shows that the inner region of the disk is disrupted by the burst, possibly being heated into a thicker, more tenuous flow, before recovering its previous form in $\sim 1000 \mathrm{~s}$. This marks the first instance that the evolution of the inner regions of an accretion disk has been observed in real-time.
\end{abstract}

Subject headings: accretion, accretion disks - line: formation - stars: individual (4U 1820-30) - stars: neutron - X-rays: binaries - X-rays: bursts

\section{Introduction}

Superbursts (e.g. Cornelisse et al. 2000; Strohmayer \& Brown 2002; Kuulkers et al. 2002) are the more powerful and rarer relations of the Type I X-ray bursts, and are thought to be due to the nuclear burning of the ashes (principally carbon and rp-process nuclei) that remain after thermonuclear processing of the accreted light elements (Strohmayer \& Brown 2002; Cumming 2003). One of the defining characteristics of a superburst is its long

\footnotetext{
${ }^{1}$ Canadian Institute for Theoretical Astrophysics, 60 St. George Street, Toronto, Ontario, Canada M5S 3H8; ballantyne@cita.utoronto.ca

${ }^{2}$ NASA Goddard Space Flight Center, Laboratory for High Energy Astrophysics, Code 680, Greenbelt, MD, USA 20771; stroh@clarence.gsfc.nasa.gov
} 
decay time of a number of hours, three orders of magnitude greater than a typical Type I burst (Kuulkers 2003). This allows high signal-to-noise spectra to be accumulated at regular intervals throughout the course of the burst.

$4 \mathrm{U} 1820-30$ is a compact low-mass X-ray binary with a period of only 11.4 minutes (Stella, Priedhorsky \& White 1987), and lies within the globular cluster NGC 6624 at a distance of $\sim 7 \mathrm{kpc}$ (Vacca, Lewin \& van Paradijs 1986; Hesser \& Shawl 1985; Rich, Minniti $\&$ Liebert 1993). Type I X-ray bursts have been observed from this system for nearly 30 years (Grindlay \& Gursky 1976). The accretion rate inferred from the persistent X-ray luminosity is $\sim 1.5 \times 10^{17} \mathrm{~g} \mathrm{~s}^{-1}$ (Cumming 2003). The first, and so far only, superburst seen from 4U 1820-30 was observed by the Rossi X-ray Timing Explorer (RXTE) on 1999 September 9 (UT) during a scheduled monitoring observation. The initial analysis and interpretation of the data was presented by Strohmayer \& Brown (2002). Time-resolved spectroscopy presented by these authors showed that an iron $\mathrm{K} \alpha$ line and absorption edge were present in the spectra, and that their properties changed over time. A likely origin for these features is reprocessing of the X-rays by the accretion disk (Day \& Done 1991). This process, called X-ray reflection, is commonly used to explain similar features in the spectra of Seyfert galaxies (e.g. Pounds et al. 1990; George \& Fabian 1991; Ballantyne, Vaughan \& Fabian 2003).

In this paper we present the results of fitting the time-resolved spectra of the superburst with detailed models of X-ray reflection. The next section describes the model calculations and details of the spectral fitting. The results are presented in Section 3 and discussed in Section 4.

\section{Model Calculations}

The X-ray reflection models were calculated with the code of Ross \& Fabian (1993) (see also Ross, Fabian \& Young 1999; Ballantyne, Iwasawa \& Fabian 2001; Ballantyne, Ross \& Fabian 2001). A one-dimensional slab of uniform density gas is illuminated by a blackbody continuum (defined from $1 \mathrm{eV}$ to $100 \mathrm{keV}$ ) of temperature $k T$, and allowed to come into thermal and ionization balance. The emergent spectrum is calculated with a Fokker-Planck/diffusion method that naturally accounts for the energy redistribution of photons by Compton scattering (Ross, Weaver \& McCray 1978). The following ions are included in the calculations: C V - VII, N VI - VIII, O V - IX, Mg IX - XIII, S XI - XV and Fe XVI - XXVII. Fluorescence and recombination lines from these ions are computed and transferred simultaneously with the continuum, resulting in a self-consistent prediction of the emitted spectrum. 
The compact orbit of this binary suggests that the donor is an evolved low-mass helium star (Rappaport et al. 1987), and thus the accretion disk material will be He-rich and H-poor (Cumming 2003). Therefore, we assumed the reflecting medium has the $\mathrm{He}, \mathrm{C}, \mathrm{N}$, and $\mathrm{O}$ abundances of cool extreme helium stars from the tabulation of Pandey et al. (2001). The $\mathrm{Mg}, \mathrm{Si}$ and $\mathrm{Fe}$ abundances were left at their solar values (Grevesse, Noels \& Sauval 1996), but the effective metallicity is increased (by $\sim 3$ ) due to the negligible levels of hydrogen. The helium is assumed to be completely ionized everywhere in the reflecting region.

The computed reflection spectra are parameterized by the ionization parameter $\xi=$ $4 \pi F_{\mathrm{X}} / n_{\mathrm{He}}$ of the irradiated gas, where $F_{\mathrm{X}}$ is the flux of the incident X-rays and $n_{\mathrm{He}}=$ $10^{15} \mathrm{~cm}^{-3}$ is the assumed number density of helium. Since the blackbody emission is also seen directly from the neutron star, the fitted spectrum is the sum of the blackbody and the reflected emission, weighted by a factor $R$, the reflection fraction. This parameter measures the relative importance of reflection in the spectrum, and can be related to the covering factor of the accretion disk.

Strohmayer \& Brown (2002) describes the RXTE data and the process of accumulating the $\sim 8064 \mathrm{~s}$ spectra during the burst. The preburst.spectrum was used as the background, and was thus subtracted from the data during spectral fitting (Strohmayer \& Brown 2002). This persistent emission (amounting to a luminosity of $1.8 \times 10^{37} \mathrm{erg} \mathrm{s}^{-1}$ ) is driven by the accretion onto the neutron star, so using this spectrum as a background implicitly assumes that the burst will not effect this underlying emission. As we see below, we cannot be sure that this does not happen; however, Strohmayer \& Brown (2002) found little difference in their spectral fits between the preburst background and the detector background. Since the flux of the superburst varies from $20 \times$ to $3 \times$ greater than the preburst emission, then the shape of the background will not greatly effect the fit results.

The spectral fitting was performed with XSPECv.11.2.0bp (Arnaud 1996). The model consisted of a grid of reflection spectra (read in using the atable command) and attenuation from neutral gas along the line of sight (using the wabs model), parameterized by the column density $N_{\mathrm{H}}$. The Galactic absorption toward $4 \mathrm{U} 1820-30$ is $1.5 \times 10^{21} \mathrm{~cm}^{-2}$ (Dickey \& Lockman 1990). The reflection spectra were blurred by the effects of relativistic motion within the Schwarzschild metric (Fabian et al. 1989). The inner radius of emission $\left(r_{\text {in }}\right)$ was allowed to vary, but the emissivity was fixed as $r^{-3}$, where $r$ is distance along the accretion disk, and the outer radius was frozen at $200 G M / c^{2}$, where $M$ is the mass of the neutron star. The inclination angle of the disk to the line of sight was fixed at $30^{\circ}$. The fitted energy range was $3-40 \mathrm{keV}$, but was changed to $3-15 \mathrm{keV} 7000 \mathrm{~s}$ into the burst because of background dominating at higher energies. The errorbars on the best fit parameters were computed using the $2 \sigma$ uncertainties for one parameter of interest. 


\section{Spectral Fitting Results}

The results of fitting the reflection models to the superburst data are shown in Figure 1. We find that an ionized reflector provides a good description to the spectra for most of the burst. During the first $1000 \mathrm{~s}$ the spectra are well fit with a large $\xi$, indicating significant ionization of the accretion disk. Over the next $\sim 500 \mathrm{~s} \xi$ decreases by more than an order of magnitude. For times between $\sim 2500$ and $3000 \mathrm{~s}$ the spectra cannot be fit by the reflection model, except for the last three points, where only very loose constraints could be placed on $\xi$. Strohmayer \& Brown (2002) showed the spectrum was hardening very rapidly during that period, which indicates that some rapid evolution of the system was ongoing, and is not well modeled by our equilibrium calculations. However, the recombination timescale for H-like $\mathrm{Fe}$ is $t_{\mathrm{recomb}} \sim 1 / n_{e} \alpha_{\mathrm{Fe}}(T)$. The slowest timescale occurs at the highest temperature, which is $10^{7} \mathrm{~K}$ at the surface of the reflector, so that $t_{\text {recomb }} \sim 10^{-4} \mathrm{~s}$, assuming the recombination coefficient of Verner \& Ferland (1996). This is the same order of magnitude as the disk dynamical time at $10 \mathrm{GM} / \mathrm{c}^{2}$. The recombination timescale of He II is $\sim 0.1 \mathrm{~s}$, much longer than the dynamical time, but faster than the $64 \mathrm{~s}$ spectra examined here. Therefore, the assumptions of ionization equilibrium or fully stripped helium cannot be the cause of the poor fits in this region of the lightcurve. A more fundamental change in the X-ray source or reflecting geometry may be required. Finally, after the last Earth occultation passage, the system was revealed to have settled into a final decaying state, with $\xi \sim 100 \mathrm{erg} \mathrm{cm} \mathrm{s}^{-1}$, although one other non-equilibrium event took place at $\sim 6000 \mathrm{~s}$.

While the disk inclination angle was fixed at $30^{\circ}$ for the spectral fits, there is a previous constraint of $35^{\circ}-50^{\circ}$ found by Anderson et al. (1997) from modulations in the ultraviolet flux (due to X-ray heating of the secondary). Relativistic disk features are also dependent on the accretion disk inclination angle (Fabian et al. 1989), so can provide a consistency check on the reflection hypothesis. In Figure 2 we plot the $68 \%, 90 \%$ and $99 \%$ confidence contours for $r_{\text {in }}$ and the disk inclination angle $5500 \mathrm{~s}$ into the superburst. This segment was chosen as it has a low reduced $\chi^{2}$ and strong reflection features. While the disk fit seems to prefer a lower inclination angle, it is consistent with the results of Anderson et al. (1997) at the $2 \sigma$ level.

The observed evolution of $\xi$ from highly to weakly ionized is to be expected since the observed flux decays over the course of the burst (Strohmayer \& Brown 2002). More fundamental insights from this analysis arise from the variations of $N_{\mathrm{H}}, R$ and $r_{\text {in }}$ over the first $2000 \mathrm{~s}$ of the superburst. The changes of both $r_{\text {in }}$, which measures the inner radius of the disk (as judged from the width of the iron line and edge), and $R$, the reflection fraction, strongly indicates that the accretion disk is significantly affected by the superburst out to a radius of $\sim 100 \mathrm{GM} / \mathrm{c}^{2}$. In addition, the line-of-sight absorbing column also went through 
a similar variation, either as a result of the burst itself or the changes to the inner accretion disk. All three of these parameters follow a similar pattern where they increase $\left(r_{\text {in }}\right.$ and $N_{\mathrm{H}}$ ) or decrease $(R)$ before returning to close to their initial value. This behavior seems to be closely linked to the blackbody temperature $k T$, rather than the flux, which decreases monotonically during the burst.

\section{Discussion}

A likely scenario to explain the observed behavior is that the accretion flow in the inner disk is altered by the superburst so that it loses the ability to produce reflection features. This could be accomplished if the scale-height of the disk, $H$, increased due to heating of the gas by the radiation field. This would decrease the surface density of the disk, allowing it to be more easily and completely ionized by the burst radiation, removing any spectral features in the observable band, and effectively becoming a mirror for the blackbody spectrum. As there would be no evidence for reprocessing from this innermost region, the estimate of the inner radius would be from further out in the disk where iron can still produce an emission line. The additional blackbody emission reflected from the ionized mirror, would dilute the reflection features, resulting in the low value of $R$. Alternatively, the lower surface density could reduce the optical depth of the gas to the point where the inner disk becomes a very inefficient reflector, while only the outer disk would be optically thick enough for efficient reprocessing.

The main evidence for this thermal effect is seen in the correlations between $r_{\text {in }}, R$ and $k T$. The Compton temperature of the gas is $k T_{\mathrm{C}} \approx k T$, and reached $\sim 3 \mathrm{keV}$, about twice the maximum disk temperature (Shakura \& Sunyaev 1973). Since $H \propto c_{\mathrm{s}} r^{3 / 2} \propto T^{1 / 2} r^{3 / 2}$, where $c_{\mathrm{s}}$ is the local sound speed, then $H$ will increase by $\sim 40 \%$ from its non-illuminated value. Changes to the disk surface density can take place over a viscous time which is $\sim 1000 \mathrm{~s}$ at $100 \mathrm{GM} / \mathrm{c}^{2}$ if $\alpha \sim 0.2$, where $\alpha$ is the Shakura-Sunyaev viscosity parameter (Shakura \& Sunyaev 1973). This timescale compares favorably with the observed changes in $R$ and $r_{\text {in }}$. An increase in $H$ may also explain the evolution of $N_{\mathrm{H}}$ as the thicker disk may bring clumps of disk material into the line-of-sight. Data which extend below the $3 \mathrm{keV}$ limit of $R X T E$ would be needed to test if the absorption was caused by ionized or neutral material. Under this hot disk scenario, once the peak in $k T$ is passed the disk material cooled to lower and lower temperatures, and thus contracted to its original size. Simultaneously, the amount of material available to reflect increased, so $R$ grew larger, but $\xi$ dropped due to a combination of the increasing surface density and the decaying burst flux.

The connection between the ionization parameter and the disk surface density $\Sigma$ can be 
formulated as $\xi \approx L_{\mathrm{X}} m_{\mathrm{He}} H / r^{2} \Sigma$, where $L_{\mathrm{X}}$ is the luminosity of the burst, and $m_{\mathrm{He}}$ is the mass of a helium nucleus. Using the equations for a gas pressure dominated Shakura-Sunyaev disk with electron scattering opacity (Shakura \& Sunyaev 1973), a peak burst luminosity of $3.4 \times 10^{38} \mathrm{erg} \mathrm{s}^{-1}$ (Strohmayer \& Brown 2002), and $\alpha=0.2$, we find $\xi \approx 100 \mathrm{erg} \mathrm{cm} \mathrm{s}^{-1}$ for $r=$ $15 \mathrm{GM} / \mathrm{c}^{2}$, which is consistent with the values of $\xi$ found near the end of the burst (Fig. 1). From the observed decay in flux (Strohmayer \& Brown 2002), the surface density of the disk must be $\sim 100$ times less than the Shakura-Sunyaev value at the start of the superburst, but only $\sim 6$ times less near the end in order to obtain the necessary level of ionization. This indicates the surface density of the inner accretion disk was significantly lower at the peak of the burst but increased as the burst decayed, supporting the interpretation that the disk material was severely affected by the explosion.

Another process that could lower the surface density at the inner edge of an accretion disk is the interaction between the radiation field and material flowing in the disk. Radiation can remove angular momentum from the disk material, causing the disk to empty inward, thus lowering the surface density. There exists only a few calculations of the interaction between an accretion disk and the radiation field from a luminous central source (Walker \& Mészáros 1989; Walker 1992; Miller \& Lamb 1993, 1996). These models predict that the radiation will exert a significant torque on the accretion flow, which increases the accretion rate close to the central object. This effect may be enhanced in the case of $4 \mathrm{U} 1820-30$ due to the power of the superburst (as compared to the typical Type I burst considered in these models), as well as an interaction between the disk and the expanding shell that was ejected from the neutron star just prior to the superburst (Strohmayer \& Brown 2002) - the radius-expansion phase (Lewin, Vacca \& Basinska 1984). It is difficult to rigorously compare this idea to the observations because detailed modeling of the disk-radiation interaction for a superburst or a radius-expansion burst has not been performed.

By the end of the observation, the accretion disk has recovered from its disruption by the burst, and is reflective down to a radius of $10-20 G M / c^{2}$, which is curiously larger than the innermost stable circular orbit of $6 G M / c^{2}$. Since this is not an X-ray pulsar, the disk would not be truncated by the star's magnetosphere. Rather, this may be evidence for the disk-radiation interaction described above, and the material within $10-20 G M / c^{2}$ is no longer dense enough to provide a reflection signature. At these late times, the fits also require strong reflection, with $R>1$, indicating that the disk covers a large solid angle from the X-ray source, and thus is thin relative to the radius of the neutron star or has a non-negligible flare at large radii. The latter possibility is supported by the reflection from $r>100 \mathrm{GM} / \mathrm{c}^{2}$ observed early in the burst. If the inner disk expanded from the X-ray heating, then a significant disk flare might be necessary in order to allow the distant material to be irradiated by the blackbody emission. 
Dipping behavior is seen in the X-ray lightcurve of $4 \mathrm{U} 1820-30$ between $6000-7500 \mathrm{~s}$ into the burst (see Fig. 7 in Strohmayer \& Brown 2002). This kind of variability in low-mass Xray binaries is often explained as variable absorption from a two-component ionized medium close to the neutron star (Frank, King \& Lasota 1987). The dipping observed from $4 \mathrm{U} 1820-$ 30 could then be due to the reformation of the accretion disk, however, the timescale is incorrect, with the dipping seen $\sim 2000 \mathrm{~s}$ after the probable recollapse of the disk. However, there is an interesting correspondence between the times when dipping is observed and an increase in $N_{\mathrm{H}}$ derived from spectral fitting (Fig. 1). This may indicate that the dipping is caused by partially ionized material that is slowly recombining, but farther away from the accretion disk. An obvious source for such material is the gas that was cast off from the neutron star during the radius expansion phase. This material could be at a large distance from the system $\left(10^{14} \mathrm{~cm}\right.$ if ejected at the escape velocity).

We thank E. Agol, L. Bildsten, E. Quataert, N. Murray \& C. Matzner for helpful discussions, and A. Fabian for providing the relativistic blurring functions. DRB is supported by the Natural Sciences and Engineering Research Council of Canada.

\section{REFERENCES}

Anderson, S.F., Margon, B., Deutsch, E.W., Downes, R.A. \& Allen, R.G., 1997, ApJ, 482, L69

Arnaud K.A., 1996, in Jacoby G., Barnes J., eds, Astronomical Data Analysis Software and Systems V, ASP Conf. Ser. Vol. 101, 17

Ballantyne, D.R., Iwasawa, K. \& Fabian, A.C., 2001, MNRAS, 323, 506

Ballantyne, D.R., Ross, R.R. \& Fabian A.C., 2001, MNRAS, 327, 10

Ballantyne, D.R., Vaughan, S. \& Fabian, A.C., 2003, MNRAS, 342, 239

Cornelisse, R., Heise, J., Kuulkers, E., Verbunt, F. \& in't Zand, J.J.M., 2000, A\&A, 357, L21

Cumming, A., 2003, ApJ, 595, 1077

Day, C.S.R. \& Done, C, 1991, MNRAS, 253, 35P

Dickey, J.M. \& Lockman, F.J., 1990, ARA\&A, 28, 215 
Fabian, A.C., Rees, M.J., Stella, L. \& White, N.E., 1989, MNRAS, 238, 729

Frank, J., King, A.R. \& Lasota, J.-P., 1987, A\&A, 178, 137

George, I.M. \& Fabian, A.C., 1991, MNRAS, 249, 352

Grevesse, N., Noels, A. \& Sauval, A.J. 1996, in Holt, S.S. \& Sonneborn, G., eds, Cosmic Abundances, ASP Conf. Ser. Vol. 99, 117

Grindlay, J. \& Gursky, H., 1996, ApJ, 205, L131

Hesser, J.E. \& Shawl, S.J., 1985, PASP, 97, 465

Kuulkers, E. et al., 2002, A\&A, 382, 503

Kuulkers, E. 2003, in van den Heuvel, E.P.J., in 't Zand, J.J.M. \& Wijers, R.A.M.J. eds, The Restless High-Energy Universe, in press (astro-ph/0310402)

Lewin, W.H.G., Vacca, W.D. \& Basinska, E.M., 1984, ApJ, 277, L57

Miller, M.C. \& Lamb, F.K., 1993, ApJ, 413, L43

Miller, M.C. \& Lamb, F.K., 1996, ApJ, 470, 1033

Pandey, G., Rao, N.K., Lambert, D.L., Jeffery, C.S. \& Asplund, M., 2001, MNRAS, 324, 937

Pounds, K.A., Nandra, K., Stewart, G.C., George, I.M. \& Fabian A.C., 1990, Nature, 344, 132

Rappaport, S., Nelson, L.A., Ma, C.P. \& Joss, P.C., 1987, ApJ, 322, 842

Rich, R.M., Minniti, S. \& Liebert, J., 1993, ApJ, 406, 489

Ross, R.R. \& Fabian, A.C., 1993, MNRAS, 261, 74

Ross, R.R., Weaver, R. \& McCray, R., 1978, ApJ, 219, 292

Ross, R.R., Fabian, A.C. \& Young, A.J., 1999, MNRAS, 306, 461

Shakura, N.I. \& Sunyaev, R.A., 1973, A\&A, 24, 337

Stella, L., Priedhorsky, W. \& White, N.E., 1987, ApJ, 312, L17

Strohmayer, T.E. \& Brown, E.F., 2002, ApJ, 566, 1045 
Vacca, W.D., Lewin, W.H.G. \& van Paradijs, J., 1986, MNRAS, 220, 339

Verner, D.A. \& Ferland, G.F., 1996, ApJS, 103, 467

Walker, M.A. \& Mészáros, P., 1989, 346, 844

Walker, M.A., 1992, ApJ, 385, 642 

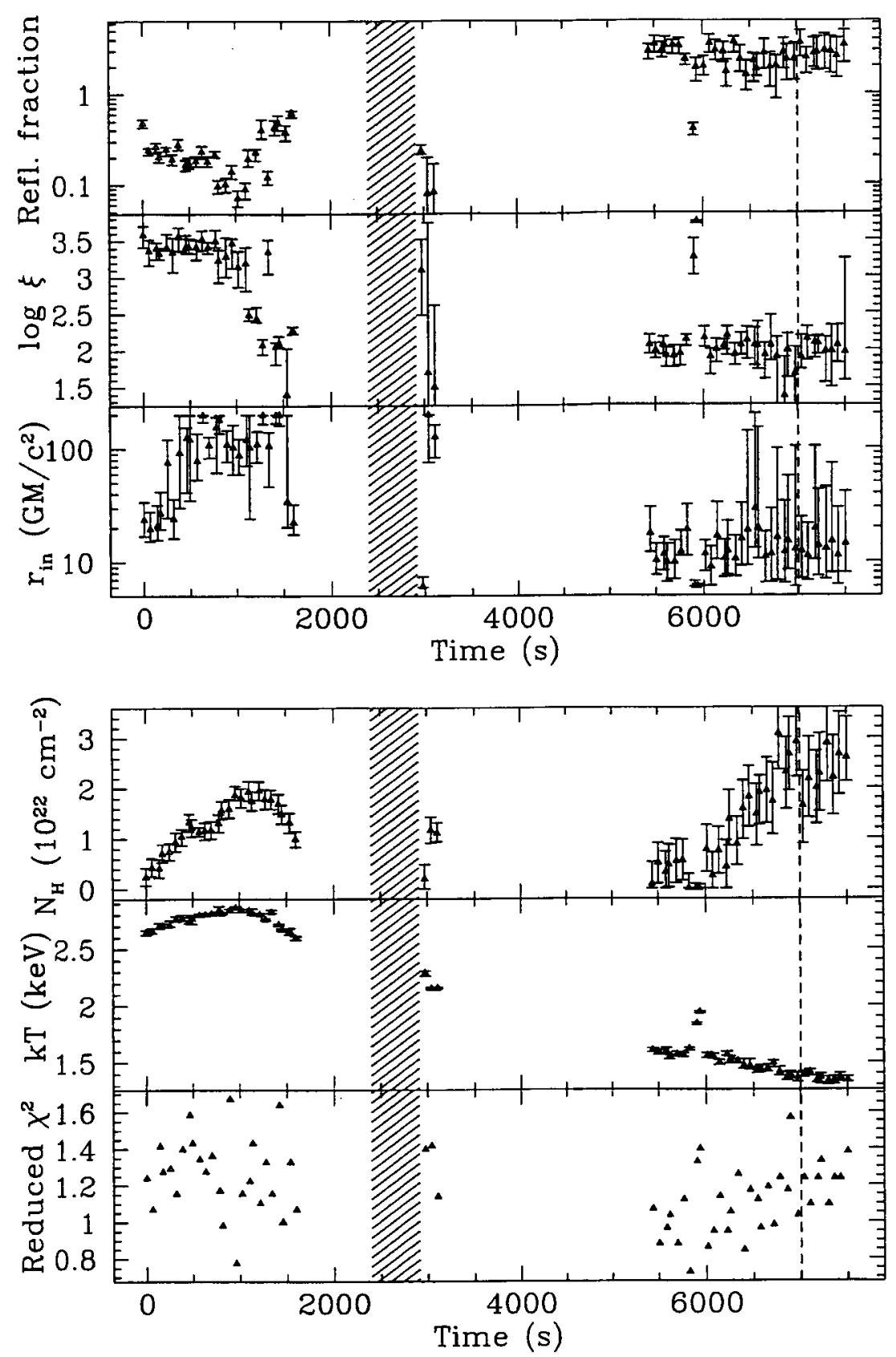

Fig. 1.- Results of fitting the time-resolved superburst spectra of $4 \mathrm{U}$ 1820-30 with ionized reflection models. The top panel shows the evolution of the reflection parameters: the ionization parameter $\xi$, the reflection fraction, and inner radius of the disk, $r_{\text {in }}$. The lower panel describes the evolution of the absorption column, $k T$ of the incident blackbody, and the reduced $\chi^{2}$ of the spectral fits. The reflection fraction, $r_{\text {in }}$, and $N_{\mathrm{H}}$ seem to be closely related to the evolution of $k T$. Data were fit from $3-40 \mathrm{keV}$ for the points to left of the dashed line, and from $3-15 \mathrm{keV}$ for the points to the right. Time equals zero corresponds to about $100 \mathrm{~s}$ after the start of the burst. The hatched region denotes the interval where the spectra were hardening rapidly, and were not well described by the equilibrium reflection model. 


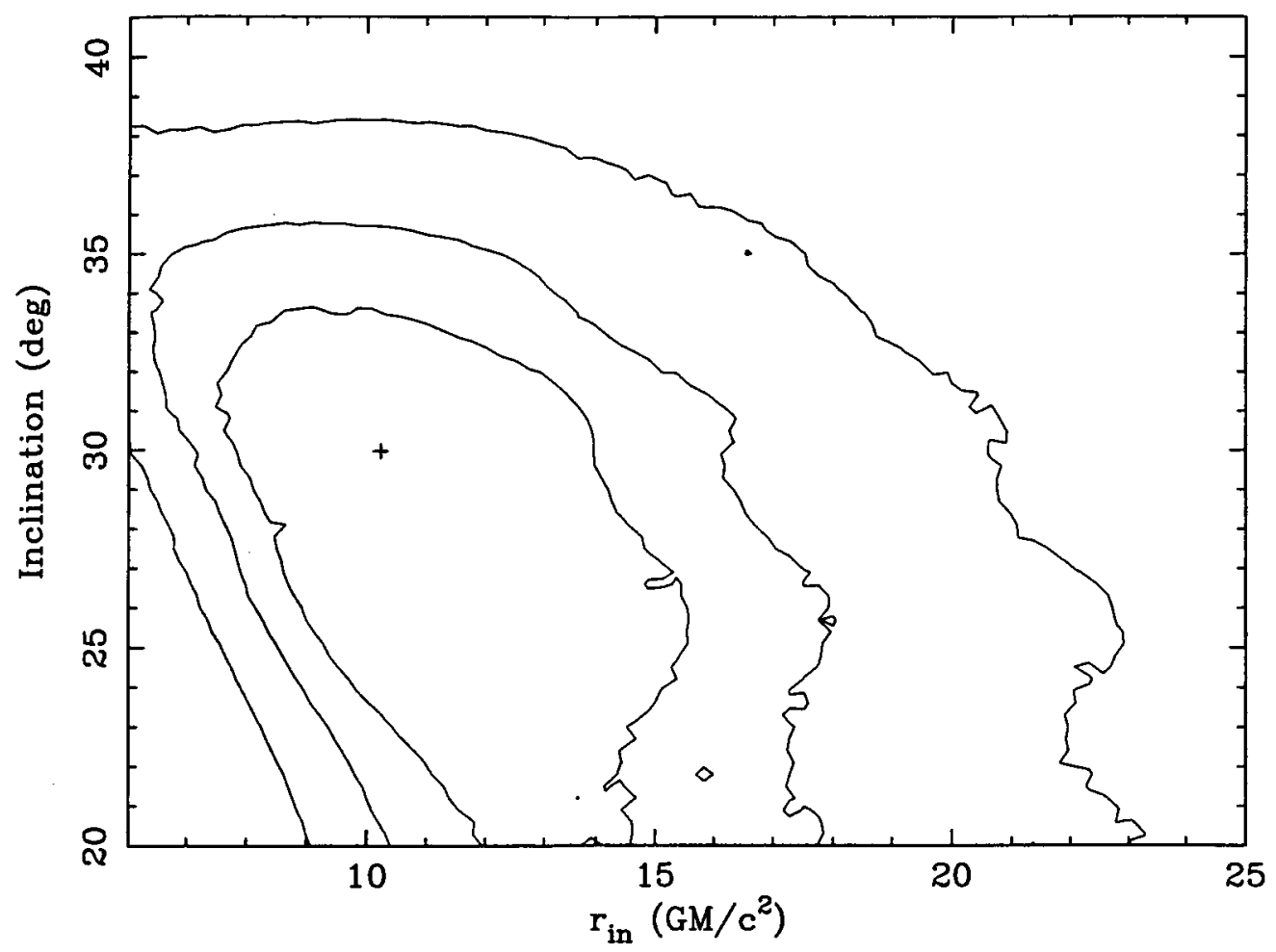

Fig. 2.- Joint $68 \%, 90 \%$ and $99 \%$ confidence contours on $r_{\text {in }}$ and the disk inclination angle for a spectral fit $5500 \mathrm{~s}$ into the superburst. Modulations in the ultraviolet flux from $4 \mathrm{U} 1820-30$ previously constrained the inclination angle to between $35^{\circ}$ and $50^{\circ}$ (Anderson et al. 1997), which is consistent with the results from disk reflection at the $2 \sigma$ level. 